International Journal of Physical Research, 9(1) (2021) 49-55
International Journal of Physical Research
SPC
Website: www.sciencepubco.com/index.php/IJPR
Research paper

\title{
Optical 1-soliton solutions of Triki-Biswas equation by the First Integral Method and the sine-cosine Method
}

\author{
S. Subhaschandra Singh* \\ Physics Department, Imphal College, Imphal-795001, Manipur, India \\ *Corresponding author E-mail:subhasic@yahoo.co.in
}

\begin{abstract}
In this paper, the First Integral Method and the Sine-Cosine Method are being used in constructing optical 1-soliton solutions of TrikiBiswas Equation that plays a vital role in the study of soliton dynamics of sub-pico-second optical pulses in mono-mode optical fibers with non-Kerr law nonlinearity and subsequently some soliton and non-soliton solutions are formally obtained.
\end{abstract}

Keywords: Optical Soliton; Triki-Biswas Equation (TBE); Optical Fibers; Non-Kerr Law Nonlinearity.

\section{Introduction}

Optical solitons are optical pulses which don't change shape during propagation because of balance between dispersion effects and nonlinear effects in the medium through which they propagate. They are also referred to as localized optical pulses which don't diffract or disperse while passing through a medium. They can act as information carriers in trans-continental and trans-oceanic data transmission. In the recent a few decades, the study of optical solitons is playing a vital role in many core areas of modern research in communication engineering. Pulse propagation in optical fibers with self-steepening(Kerr Dispersion) is governed by the so-called Derivative Nonlinear Schrodinger Equation (DNLSE) [1] including the Kaup-Newell Equation (KNE) [2], [3], the Chen-Lee-Liu Equation (CLLE) [4], [5],the Fokas-Lenells Equation (FLE) [ 6-8], the Gerdjikov-Ivanov Equation (GIE) [9], [10] etc. As an important generalization of the derivative nonlinear Schrodinger equation, HouriaTriki andAnjan Biswas, in the year 2018, proposed an equation latter known after them as TrikiBiswas Equation (TBE) [11-13], in order to model ultra-short pulse propagation in optical fiber systems beyond the Kerr limit. The equation then becomes an excellent asset in telecommunication engineering via optical solitons. In this paper, the TBE is being handled by the aid of the first integral method [ 14-21] and the sine-cosine method [22], [23].

\section{Governing equation}

The Triki-Biswas equation (TBE) is written in the form

$$
i \frac{\partial u}{\partial t}+\alpha \frac{\partial^{2} u}{\partial x^{2}}+i \beta \frac{\partial}{\partial x}\left(|u|^{2 \mathrm{n}} u\right)=0
$$

where $\mathrm{i}=\sqrt{-1}$ is the imaginary number, $x$ is the spatial co-ordinate, $t$ is the temporal co-ordinate, the function $u(x, t)$ is a complex valued function that can yield the wave profile, the first term corresponds to a temporal evolution of pulses, the second term represents a Group Velocity Dispersion (GVD) term with coefficient $\alpha$ and the third term represents a non-Kerr and nonlinear dispersion (NLD) term compensated by the coefficient $\beta$ when $n>0$. When $n=1$, the equation reduces to Kaup-Newell equation (KNE). For $n=2$, the derivative quintic non-Kerr nonlinearity term plays an important role in the propagation of very short pulses of width around sub-10 fs in highly nonlinear optical fibers.

To reduce Eq. (1) to a nonlinear ODE, let us introduce the transformations

$$
u(x, t)=U(\xi) \mathrm{e}^{i(-k x+\omega t+\varepsilon)}
$$

and

$$
\xi=x-c t
$$

where $\mathrm{c}$ is a constant (generally the constant propagation speed of the optical pulse), $\mathrm{k}$ is the angular frequency and $\omega$ is the wave number of the pulse. 
Substituting Eqs.(2) and (3) along with the relevant derivatives into Eq. (1) and then separating the resulting equation into the real and the imaginary parts, we obtain the Real Part as

$$
\alpha \frac{d^{2} U}{d \xi^{2}}-\left(\alpha k^{2}+\omega\right) U+\beta k U^{2 n+1}=0
$$

and the Imaginary Part as

$$
c=-2 a k+(2 n+1) \beta U^{2 n}
$$

Here, we see that the speed of the sub-10 fs optical pulse (soliton) is yielded from the imaginary component shown in Eq. (5)whereas the profile of the soliton is given by integration of the real component shown in Eq. (4).

Further, let us put

$$
U(\xi)=[V(\xi)]^{N}
$$

where $N$ is to be determined from balancing of degrees between the term with highest order derivative and the term with highest nonlinearity in Eq. (4).

Thus, we have

$N+2=(2 n+1) N$ giving $N=\frac{1}{n}$

Thus, Eq. (6) becomes

$U(\xi)=[V(\xi)]^{\frac{1}{n}}$

Substituting Eq. (7) and its relevant derivatives into Eq. (4), we obtain

$2 n \alpha V V^{\prime \prime}+\alpha(1-2 n)\left(V^{\prime}\right)^{2}-4 n^{2}\left(\alpha k^{2}+\omega\right) V^{2}+4 n^{2} \beta k V^{3}=0$

where $V^{\prime}=\frac{d V}{d \xi}, V^{\prime \prime}=\frac{d^{2} V}{d \xi^{2}}$.

If we solve $\mathbf{V}(\xi)$ from Eq. (8), then using Eqs. (2), (3) and (6), we can obtain the solution of Eq. (1).

\section{Algorithm of the first integral method}

The First Integral Method, which is based on the ring theory of commutative algebra, was first proposed by Feng [14] and it was further developed by himself [15-17]. The method was then used by many researchers in finding exact solutions of different types of nonlinear evolution equations (NLEEs) which arise in many fields of science and engineering. The algorithm of it is summarized as in the following.

We consider a nonlinear partial differential equation (NLPDE) in the form

$F\left(u, u_{x}, u_{t}, u_{x x}, u_{x t}, u_{t t}, \ldots\right)=0$

where $F$ is a polynomial in the function $u(x, t)$ and its various partial derivatives while the subscripts indicate partial derivatives with respect to them.

Introducing the transformations

$u(x, t)=u(\xi), \xi=x-c t$

where $\mathrm{c}$ is a constant (generally the constant speed of wave propagation), Eq. (9) is reduced to a nonlinear ordinary differential equation (NLODE) of the form

$$
G\left(U, U^{\prime}, U^{\prime \prime}, U^{\prime \prime \prime}, \ldots\right)=0
$$

where $U^{\prime}=\frac{d U}{d \xi}, U^{\prime \prime}=\frac{d^{2} U}{d \xi^{2}}$,etc. and $G$ is a polynomial in $U$ and its derivatives.

If all terms in Eq.(11) contain derivatives, it is to be integrated until at least one term does not contain derivative and the constant of integration is to be chosen as zero in each step.

Let us assume that the solution of the reduced NLODE (11) or its integrated form has the form

$$
S(\xi)=U(\xi)
$$

and let us introduce a new independent variable $Y=Y(\xi)$ such that

$$
Y(\xi)=S^{\prime}(\xi)=\frac{d S}{d \xi}=U^{\prime}(\xi)=\frac{d U}{d \xi} .
$$

Thus, we write a plane autonomous system 


$$
\left\{\begin{array}{c}
S^{\prime}(\xi)=Y(\xi) \\
Y^{\prime}(\xi)=H(S(\xi), Y(\xi))
\end{array}\right.
$$

where $H$ is a polynomial in $S(\xi)$ and $Y(\xi)$.

If we can find two first integrals to the system of Eqs. (14) under the same conditions, then analytic solutions of system (14) can be directly obtained. However, in general, it is really difficult for us to realize this even for one first integral. Because, for a given plane autonomous system, there exists neither a systematic theory that can tell one how to find such first integrals nor a logical way for telling one what these first integrals are. We have to apply the Division Theorem of commutative algebra to obtain one first integral to the system of Eqs. (14) that will reduce Eq. (11) to a first order integrable ODE. An exact solution of Eq. (9) can be obtained by solving this ODE. For convenience, we recall the division theorem for two variables in the complex domain $C[w, z]$.

\section{DivisionTheorem:}

For two polynomials $P(w, z)$ and $Q(w, z)$ in a complex domain $C[w, z]$, if $P(w, z)$ is irreducible in $C[w, z]$ and if $Q(w, z)$ vanishes at all zero points of $P(w, z)$, then there exists another polynomial $R(w, z)$ in $C[w, z]$ such that $Q(w, z)=P(w, z) R(w, z)$. The division theorem follows immediately from Hilbert-Nullstellansatz theorem of commutative algebra.

\section{Application of the first integral method to triki-biswas equation}

Substituting $S(\xi)=V(\xi)$ and $Y(\xi)=V^{\prime}(\xi)$ into Eq. (8), we obtain the plane autonomous system

$$
\left\{\begin{array}{l}
Y(\xi)=S^{\prime}(\xi)=\frac{d S}{d \xi}, \\
Y^{\prime}(\xi)=\frac{d Y}{d \xi}=\left(1-\frac{1}{2 n}\right) \frac{Y^{2}}{S}+\frac{2 n}{\alpha}\left(\alpha k^{2}+\omega\right) S-\frac{2 n \beta k}{\alpha} S^{2} .
\end{array}\right.
$$

Let us apply the transformation

$$
\frac{d \xi}{d \eta}=S(\text { where } \eta \text { is a newvariable })
$$

to reduce Eq. (15) to

$$
\left\{\begin{array}{l}
\frac{d S}{d \eta}=S Y, \\
\frac{d Y}{d \eta}=\left(1-\frac{1}{2 n}\right) Y^{2}+\frac{2 n}{\alpha}\left(\alpha k^{2}+\omega\right) S^{2}-\frac{2 n \beta k}{\alpha} S^{3} .
\end{array}\right.
$$

We assume that $S(\eta)$ and $Y(\eta)$ are nontrivial solutions of Eq. (17) and

$Q(S(\eta), Y(\eta))=\sum_{j=0}^{m} a_{j}(S) Y^{j}(S)$

is an irreducible polynomial in the complex domain $C[S, Y]$ such that

$$
Q(S(\eta), Y(\eta))=\sum_{j=0}^{m} a_{j}(S) Y^{j}(S)=0
$$

where $a_{j}(S)(j=0,1,2,3, \ldots m)$ are polynomials in $S$ and $a_{m} \neq 0$.

Eq. (18) is the first integral of the system of equations (17).

By division theorem, there exists a polynomial $g(S)+h(S) Y$ in the complex domain $C[S, Y]$ such that

$$
\frac{d Q}{d \eta}=\frac{d Q}{d S} \frac{d S}{d \eta}+\frac{d Q}{d Y} \frac{d Y}{d \eta}=\{g(S)+h(S) Y\} \sum_{j=0}^{m} a_{j}(S) Y^{j} .
$$

Now, using Eqs.(17), (18) and (19), we obtain

$$
\begin{aligned}
& \sum_{j=0}^{m} a_{j}^{\prime}(S) S Y^{j+1}+\sum_{j=0}^{m} j a_{j}(S) Y^{j-1}\left\{\left(1-\frac{1}{2 n}\right) Y^{2}+\frac{2 n}{\alpha}\left(\alpha k^{2}+\omega\right) S^{2}-\frac{2 n \beta k}{\alpha} S^{3}\right\} \\
& \quad=\sum_{j=0}^{m} g(S) a_{j}(S) Y^{j}+\sum_{j=0}^{m} h(S) a_{j}(S) Y^{j+1} .
\end{aligned}
$$

From Eq. (20), equating coefficients of $Y^{j}(j=m+1, m \ldots 3,2,1,0)$ from both sides, we obtain

$$
\begin{aligned}
& S a_{m}^{\prime}(S)=h(S) a_{m}(S)-m\left(1-\frac{1}{2 n}\right) a_{m}(S) \\
& \left.S a_{m-1}^{\prime}(S)=g(S) a_{m}(S)+h(S) a_{m-1}(S)-(m-1)\left(1-\frac{1}{2 n}\right) a_{m-1}(S),\right) \\
& \ldots=\ldots \text { etc. } \\
& S a_{2}^{\prime}(S)=g(S) a_{3}(S)+h(S) a_{2}(S)-2\left(1-\frac{1}{2 n}\right) a_{2}(S)-4 a_{4}(S)\left\{\frac{2 n}{\alpha}\left(\alpha k^{2}+\omega\right) S^{2}-\frac{2 n \beta k}{\alpha} S^{3}\right\}
\end{aligned}
$$




$$
\begin{aligned}
& S a_{1}^{\prime}(S)=g(S) a_{2}(S)+h(S) a_{1}(S)-\left(1-\frac{1}{2 n}\right) a_{1}(S)-3 a_{3}(S)\left\{\frac{2 n}{\alpha}\left(\alpha k^{2}+\omega\right) S^{2}-\frac{2 n \beta k}{\alpha} S^{3}\right\}, \\
& S a_{0}^{\prime}(S)=g(S) a_{1}(S)+h(S) a_{0}(S)-2 a_{2}(S)\left\{\frac{2 n}{\alpha}\left(\alpha k^{2}+\omega\right) S^{2}-\frac{2 n \beta k}{\alpha} S^{3}\right\} \\
& a_{1}(S)\left\{\frac{2 n}{\alpha}\left(\alpha k^{2}+\omega\right) S^{2}-\frac{2 n \beta k}{\alpha} S^{3}\right\}=g(S) a_{0}(S) .
\end{aligned}
$$

Since $a_{m}(S)$ is a polynomial in $\mathrm{S}$, we deduce from Eq.(21a) that

$$
h(S)=m\left(1-\frac{1}{2 n}\right)
$$

For simplicity, we choose $a_{m}(S)=1$. Then, we can find the degrees of $g(S), a_{1}(S), a_{2}(S)$, etc. by balancing of degrees in equations (21). Thus, we can express these functions as polynomials of proper degrees in $S$ with undetermined coefficients. Substituting such polynomials in appropriate equations given in the system (21) and then equating coefficients of like powers of $S$ from both sides of each of the resulting equations, we can determine the unknown coefficients. Subsequently, we can know the exact expressions for $g(S), a_{0}(S), a_{1}(S), a_{2}(S)$, etc.

Substitution of these expressions into Eq.(18) can yield the expression(s) for $Y$ and using the relation $Y=\frac{d S}{d \xi}=\frac{d V}{d \xi}$, we can obtain $S(\xi) \operatorname{orV}(\xi)$ through integration. Now, using Eqs. (2), (3) and (6), we can obtain $u(x, t)$.

For $m=1$ :

Taking $m=1$, the system (21) yields

$$
\begin{aligned}
& S a_{1}^{\prime}(S)=\left\{h(S)-\left(1-\frac{1}{2 n}\right)\right\} a_{1}(S), \\
& S a_{0}^{\prime}(S)=g(S) a_{1}(S)+h(S) a_{0}(S),
\end{aligned}
$$

and

$a_{1}(S)\left\{\frac{2 n}{\alpha}\left(\alpha k^{2}+\omega\right) S^{2}-\frac{2 n \beta k}{\alpha} S^{3}\right\}=g(S) a_{0}(S)$.

As $a_{j}(S)(j=0,1)$ are polynomials, Eq. (23a) suggests that $a_{1}(S)=$ constant and

$$
h(S)=1-\frac{1}{2 n}
$$

For simplicity, we take $a_{1}(S)=1$. Then, balancing of degrees suggests that

degree $[g(S)]=1$ and degree $\left[a_{0}(S)\right]=2$.

Let us assume that

$g(S)=A_{0}+A_{1} S$

and

$a_{0}(S)=B_{0}+B_{1} S+B_{2} S^{2}$

where $A_{0}, A_{1}, B_{0}, B_{1}$ and $B_{2}$ are arbitrary constants to be determined latter.

Substituting Eqs.(25) and (26) into Eq. (23b) and equating coefficients of like powers of $S$ from both sides, we obtain

$A_{0}=\left(\frac{1}{2 n}-1\right) B_{0}$ and $A_{1}=\frac{1}{2 n} B_{1}$

Using Eq. (27) in Eq. (25), we write

$g(S)=\left(\frac{1}{2 n}-1\right) B_{0}+\frac{1}{2 n} B_{1} S$

Substituting the expressions for $a_{0}(S), a_{1}(S)$ and $g(S)$ into Eq.(23c) and equating coefficients of like powers of $S$ from both sides, we obtain

$$
\begin{aligned}
& \left(\frac{1}{2 n}-1\right) B_{0}^{2}=0, \\
& \left(\frac{1}{n}-1\right) B_{0} B_{1}=0, \\
& \left(\frac{1}{2 n}-1\right) B_{0} B_{2}+\frac{1}{2 n} B_{1}^{2}=\frac{2 n}{\alpha}\left(\alpha k^{2}+\omega\right),
\end{aligned}
$$




$$
\frac{1}{2 n} B_{1} B_{2}=-\frac{2 n \beta k}{\alpha} .
$$

Solving the above system of Eqs. (29), we obtain

$$
B_{0}=0, B_{1}= \pm 2 n \sqrt{\frac{\alpha k^{2}+\omega}{\alpha}}, B_{2}=\mp 2 n \beta k \sqrt{\frac{1}{\alpha\left(\alpha k^{2}+\omega\right)}}
$$

Substituting these values of $B_{0}, B_{1}$ and $B_{2}$ into Eq. (26), we obtain

$$
a_{0}(S)= \pm 2 n \sqrt{\frac{\alpha k^{2}+\omega}{\alpha}} S \mp 2 n \beta k \sqrt{\frac{1}{\alpha\left(\alpha k^{2}+\omega\right)}} S^{2} \text {. }
$$

Substituting the values of $a_{0}(S)$ and $a_{1}(S)$ into Eq. (18), we obtain

$$
Y=\frac{d S}{d \xi}=\mp 2 n \sqrt{\frac{\alpha k^{2}+\omega}{\alpha}} S \pm 2 n \beta k \sqrt{\frac{1}{\alpha\left(\alpha k^{2}+\omega\right)}} S^{2} .
$$

Eq. (32) has the solutions

$S(\xi)=V(\xi)= \pm \frac{\left(\alpha k^{2}+\omega\right)}{2 \beta k}\left\{1 \pm \tanh \left(n \sqrt{\frac{\alpha k^{2}+\omega}{\alpha}} \xi\right)\right\}$

and

$$
S(\xi)=V(\xi)= \pm \frac{\left(\alpha k^{2}+\omega\right)}{2 \beta k}\left\{1 \pm \operatorname{coth}\left(n \sqrt{\frac{\alpha k^{2}+\omega}{\alpha}} \xi\right)\right\}
$$

Using Eqs. (2), (7) and (33), we write the soliton solutions of Eq. (1) as

$$
u(x, t)=\left( \pm \frac{\alpha k^{2}+\omega}{2 \beta k}\right)^{\frac{1}{n}}\left[1 \pm \tanh \left\{n \sqrt{\frac{\alpha k^{2}+\omega}{\alpha}}(x-c t)\right\}\right]^{\frac{1}{n}} e^{i(-k x+\omega t+\varepsilon)}
$$

and

$u(x, t)=\left( \pm \frac{\alpha k^{2}+\omega}{2 \beta k}\right)^{\frac{1}{n}}\left[1 \pm \operatorname{coth}\left\{n \sqrt{\frac{\alpha k^{2}+\omega}{\alpha}}(x-c t)\right\}\right]^{\frac{1}{n}} e^{i(-k x+\omega t+\varepsilon)}$

with the validity condition $\alpha\left(\alpha k^{2}+\omega\right)>0$.

Here, Eq. (34a) represents a dark optical 1-soliton solution and Eq. (34b) represents a singular optical 1-soliton solution.

One can try for more solutions with $m=2,3,4$ which will become complicated. Attempts for solutions with $m \geq 5$ can be dropped as algebraic equations with degrees greater than or equal to 5 are generally not solvable.

\section{Algorithm of sine-cosine method}

We set the solution of Eq.(11) (or its integrated version, if necessary) as

$$
U(\xi)=A \sin ^{p}(\mu \xi),|\xi| \leq \frac{\pi}{\mu}
$$

or in the form

$$
U(\xi)=A \cos ^{p}(\mu \xi),|\xi| \leq \frac{\pi}{2 \mu},
$$

where $A, p, \mu$ are parameters to be determined latter.

If we choose the solution in the form of Eq. (35), we have

$$
\begin{aligned}
U(\xi) & =A \sin ^{p}(\mu \xi) \\
U^{m}(\xi) & =A^{m} \sin ^{m p}(\mu \xi) \\
\left(U^{m}\right)_{\xi} & =\mu m p A^{m} \sin ^{m p-1}(\mu \xi) \cos (\mu \xi) \\
\left(U^{m}\right)_{\xi \xi} & =-\mu^{2} m^{2} p^{2} A^{m} \sin ^{m p}(\mu \xi)+m p(m p-1) \mu^{2} A^{m} \sin ^{m p-2}(\mu \xi), \text { etc. }
\end{aligned}
$$


and if we choose the solution in the form of Eq. (36), we have

$$
\begin{aligned}
U(\xi) & =A \cos ^{p}(\mu \xi), \\
U^{m}(\xi) & =A^{m} \cos ^{m p}(\mu \xi), \\
\left(U^{m}\right)_{\xi} & =-\mu m p A^{m} \cos ^{m p-1}(\mu \xi) \sin (\mu \xi), \\
\left(U^{m}\right)_{\xi \xi} & =-\mu^{2} m^{2} p^{2} A^{m} \cos ^{m p}(\mu \xi)+m p(m p-1) \mu^{2} A^{m} \cos ^{m p-2}(\mu \xi), \text { etc. }
\end{aligned}
$$

Substituting Eqs. (37) or (38) into the reduced equation i. e Eq. (11) or its integrated-out version, as the case may be, we balance the exponents of suitable pair(s) of sine functions (if Eqs. (37) are used) or of the cosine functions (if Eqs. (38) are used). Then, we are to collect the coefficients of like powers of sine or cosine functions and to set them to zero. Thus, we will obtain a system of algebraic equations. Solving such a system of algebraic equations manually or with the help of a suitable computer software like Maple or Mathematica, we can obtain the values of the parameters $A, p$ and $\mu$. Substituting the values of these parameters into Eq. (35) or (36), as the case may be, we can obtain travelling wave solutions of Eq. (11) and hence of Eq. (9).

\section{Application of the sine-cosine method to Triki-Biswas equation}

The algorithm stated in the previous section is being used in finding solutions of Eq. (4).

Choosing the solution of Eq. (4) in the form of Eqs. (35) and substituting Eqs. (37) into Eq. (4), we obtain

$$
-\alpha \mu^{2} p^{2} A \sin ^{p}(\mu \xi)+\alpha p(p-1) \mu^{2} A \sin ^{p-2}(\mu \xi)-\left(\alpha k^{2}+\omega\right) A \sin ^{p}(\mu \xi)+\beta k A^{2 n+1} \sin ^{p(2 n+1)}(\mu \xi)=0 .
$$

From Eq. (39), balancing the exponents from $\sin ^{p-2}(\mu \xi)$ and $\sin ^{p(2 n+1)}(\mu \xi)$, we obtain

$p-2=p(2 n+1)$, i. e., $p=-\frac{1}{n}$.

With this value of $p$, Eq. (39) becomes

$-\frac{\mu^{2}}{n^{2}} \alpha A \sin ^{-\frac{1}{n}}(\mu \xi)+\frac{\alpha}{n}\left(\frac{1}{n}+1\right) \mu^{2} A \sin ^{-\frac{1}{n}-2}(\mu \xi)-\left(\alpha k^{2}+\omega\right) A \sin ^{-\frac{1}{n}}(\mu \xi)+\beta k A^{2 n+1} \sin ^{-\frac{1}{n}-2}(\mu \xi)=0$.

From Eq. (40), we obtain

$\frac{\alpha \mu^{2}}{n^{2}}=-\left(\alpha k^{2}+\omega\right)$

giving

$$
\mu= \pm n \sqrt{-\frac{\left(\alpha k^{2}+\omega\right)}{\alpha}}
$$

and

$$
\beta k A^{2 n}=-\frac{\alpha(1+n) \mu^{2}}{n^{2}}
$$

giving

$$
A=\left[\frac{(1+n)\left(\alpha k^{2}+\omega\right)}{\beta k}\right]^{\frac{1}{2 n}} .
$$

Substituting the values of $A, p$ and $\mu$ into Eq. (35), we obtain the solution of Eq. (4) as

$$
U(\xi)= \pm\left[\frac{(1+n)\left(\alpha k^{2}+\omega\right)}{\beta k}\right]^{\frac{1}{2 n}} \sin ^{-\frac{1}{n}}\left\{n \sqrt{-\frac{\left(\alpha k^{2}+\omega\right)}{\alpha}} \xi\right\}= \pm i\left[\frac{(1+n)\left(\alpha k^{2}+\omega\right)}{\beta k}\right]^{\frac{1}{2 n}} \operatorname{cosech}^{\frac{1}{n}}\left\{n \sqrt{\frac{\left(\alpha k^{2}+\omega\right)}{\alpha}} \xi\right\}
$$

Similarly, choosing the solution in the form of Eq. (36), we obtain the solution of Eq. (4) as

$$
U(\xi)= \pm\left[\frac{(1+n)\left(\alpha k^{2}+\omega\right)}{\beta k}\right]^{\frac{1}{2 n}} \operatorname{sech}^{\frac{1}{n}}\left\{n \sqrt{\frac{\left(\alpha k^{2}+\omega\right)}{\alpha}} \xi\right\}
$$

Using Eqs. (2), (3) and (42), we obtain soliton solutions of Eq. (1) as

$$
u(x, t)= \pm i\left[\frac{(1+n)\left(\alpha k^{2}+\omega\right)}{\beta k}\right]^{\frac{1}{2 n}} \operatorname{cosech}^{\frac{1}{n}}\left\{n \sqrt{\frac{\left(\alpha k^{2}+\omega\right)}{\alpha}}(x-c t)\right\} e^{i(-k x+\omega t+\varepsilon)}
$$




$$
u(x, t)= \pm\left[\frac{(1+n)\left(\alpha k^{2}+\omega\right)}{\beta k}\right]^{\frac{1}{2 n}} \operatorname{sech}^{\frac{1}{n}}\left\{n \sqrt{\frac{\left(\alpha k^{2}+\omega\right)}{\alpha}}(x-c t)\right\} e^{i(-k x+\omega t+\varepsilon)}
$$

Here, Eq. (43) represents a singular 1-soliton solution and Eq. (44) represents a bright 1-soliton solution with the same validity condition $\alpha\left(\alpha k^{2}+\omega\right)>0$.

\section{Conclusion}

In the present paper, bright, dark and singular optical 1-soliton solutions of Triki-Biswas equation, that often arises in the investigation of the dynamics of optical soliton propagation through optical fibers, are obtained via two methods namely the First Integral Method and the Sine-Cosine Method. These two methods are powerful and efficient techniques for finding exact optical soliton solutions for a wide variety of nonlinear evolution equations (NLEEs) arising in many areas of nonlinear science and engineering.

\section{Conflict of interest}

There is no conflict of interest.

\section{Funding source}

There is no Funding Source for the work.

\section{References}

[1] H. Triki, A. Biswas, Sub-picosecond chirped envelope solitons and conservation laws in mono-mode optical fibers for a new derivative nonlinear Schrodinger's model, Optik 173 pp. 235-241, 2018.https://doi.org/10.1016/i.ijleo.2018.08.026.

[2] D. I. Kaup, A. C. Newell, An exact solution for a derivative nonlinear Schrodinger equation, J. Math. Phys. 19 pp. 798-801, 1978. https://doi.org/10.1063/1.523737.

[3] A. Biswas, Y. Yildrim, E. Yasar, Q. Zhou, S. P. Moshokoa, M. Belic, Sub-picosecond pulses in mono-mode optical fibers with Kaup-Newell equation by a couple of integrationschemes, Optik 167, pp. 121-128, 2018.https://doi.org/10.1016/j.ijleo.2018.04.063.

[4] H. H. Chen, Y. C. Lee, C. S. Liu, Integrability of nonlinear Hamiltonian systems by inverse scattering method, Phys. Scr. 20 pp. $490-492$, 1979.https://doi.org/10.1088/0031-8949/20/3-4/026.

[5] A. S. H. F. Mohammed, H. O. Bakodah, M. Manaja, A. Alshaery, Q. Zhou, A. Biswas, S.Moshokoa, M. R. Belic, Bright optical solitons of ChenLee-Liu equation with improved Adomian decomposition method, Optik 181, pp. 964-970, 2019.https://doi.org/10.1016/j.ijleo.2018.12.177.

[6] J. Xu, E. Fan, Long-time asymptotics for the Fokas-Lenells equation with decaying initial value problem without solitons, J. Differ. Equ. 259, pp.1098-1148, 2015. https://doi.org/10.1016/j.jde.2015.02.046.

[7] A. Biswas, Y. Yildrim, E. Yasar, Q. Zhou, S. P. Mosokoa, M. R. Belic, Optical soliton solutions to Fokas-Lenells equation using some different methods, Optik 173, pp. 21-31, 2018.https://doi.org/10.1016/j.ijleo.2018.07.098.

[8] M. Arshed, D. Lu, Mutti-Ur Rehman, I. Ahmed, A. M. Sultan, Optical solitary wave and elliptic function solutions of the Fokas-Lenells equation in the presence of perturbation terms and modulation stability, Phys. Scr. 94(10), Article ID 105202, 2019.https://doi.org/10.1088/14024896/ab1791.

[9] H. Yilmaz, Exact solutions of the Gerdjikov-Ivanov equation using Darboux transformations, J. Nonlinear Math. Phys 22(1), pp. 32-46, 2015.https://doi.org/10.1080/14029251.2015.996438.

[10] N. Kedkhoda, H. Jfari, Analytical solutions of Gerdjikov-Ivanov equation by using expifo:(- $\phi(z))$-expansion, Optik 139, pp. 72-76, 2017.https://doi.org/10.1016/j.ijleo.2017.03.078.

[11] N. A. Kudryashov, First integrals and solutions of the travelling wave reduction for the Triki-Biswas equation, Optik 183, pp. 275-281, 2019.https://doi.org/10.1016/j.ijleo.2019.03.087.

[12] Y. Yildrim, Sub-pico-second pulses in mono-mode optical fibers with Triki-Biswas model using trial equation architecture, Optik 183, pp. 463466, 2019.https://doi.org/10.1016/j.ijleo.2019.02.018.

[13] A. I. Aliyu, A. S. Alshomrani, M. Inc, D. Baleanu, Optical solitons for Triki-Biswas equation by two analytic approaches, AIMS Math. 5(2), pp. 1001-1010, 2020.https://doi.org/10.3934/math.2020069.

[14] Z.S. Feng, The first integral method to study the Burgers Korteweg-de Vries equation, J. Phys. A, 35 (2), pp. $343-349$, 2002.https://doi.org/10.1088/0305-4470/35/2/312.

[15] Z.S. Feng, On explicit exact solutions to the compound Burg ers- Korteweg-de Vries equation, Phys. Lett. A. 293, pp. 57 - 66, 2002.https://doi.org/10.1016/S0375-9601(01)00825-8.

[16] Z. S. Feng, Travelling wave behavior for a generalized Fisher equation, Chaos, Soliton.Fract., 38, pp.481 - 488, 2008.https://doi.org/10.1016/j.chaos.2006.11.031.

[17] A. E. Achab, A. Bekir, Travelling Wave Solutions to the Generalized Benjamin-Bona-Mahony (BBM)Equation Using the First Integral Method, Int. J. Nonlinear Sci. 19(1), pp. 40-46, 2015.

[18] ] E. M. E. Zayed, Y. A. Amer, The First Integral Method and its Application for Deriving the Exact Solutions of a Higher-Order Dispersive CubicQuintic Nonlinear Schrodinger Equation, Comput. Math. Model 27(1), pp. 80-94, 2016.https://doi.org/10.1007/s10598-015-9305-y.

[19] S. Subhaschandra Singh, Soliton solutions of a generalized Klein-Gordon equation with power-law nonlinearity via the first integral method, Int. J. Math. Phys. 9(2), pp. 116-121, 2018.https://doi.org/10.26577/ijmph.2018.v9i2.268.

[20] F. L. Hasan, First Integral Method for Constructing New Exact Solutions of the Important Nonlinear Evolution Equations in Physics, J. Phys.: Conf. Ser. 1530 012109, 2020.https://doi.org/10.1088/1742-6596/1530/1/012109.

[21] Q. Zhang, M. Xiong, L. Chen, Exact solutions of two nonlinear partial differential equations by the first integral method, Adv. Pure Math., 10(1), pp. 12-20, 2020.https://doi.org/10.4236/apm.2020.101002.

[22] A. M. Wazwaz, A Sine-Cosine Method for Handling Nonlinear Wave Equations, Math. Comput. Model. 40, pp. 499-508, 2004.https://doi.org/10.1016/j.mcm.2003.12.010.

[23] S. S. Singh, Bright and Singular Optical 1-soliton Solutions of Lakshmanan-Porsezian-DanielEquationwith Kerr-law Nonlinearity by Sine-Cosine Method, Int. J. Sci. Res. Phys. Appl. Sci. 8(5), pp. 12-16, 2020. 